\title{
Geraten Fortpflanzungsmedizin und Stammzellenforschung in der Schweiz ausser Kontrolle?
}

\author{
N. Zwicky-Aeberhard ${ }^{a}$, U. Kayserb ${ }^{b}$ R. Graf
}

a Präsident der Vereinigung Katholischer Ärzte der Schweiz (VKAS)

b Präsident der Arbeitsgruppe für Bioethik der Schweizer Bischofskonferenz

Korrespondenz:

Dr. med. Nikolaus Zwicky-Aeberhard Hünibachstrasse 56

CH-3626 Hünibach

Tel. 0332222256

Fax 0332220156

E-Mail: info@medcath.ch

Dr. med. Urs Kayser

Oberseemattweg 4

CH-6403 Küssnacht am Rigi

Tel. 0418543060

Fax 0418543065

E-Mail: urs.kayser@bluewin.ch

Dr. theol. Roland Graf

Hertistrasse 1

CH-8842 Unteriberg

Tel./Fax 0554142970

E-Mail: webmaster@cloning.ch
Die Bundesbehörden haben das Fortpflanzungsmedizingesetz (FMedG) bisher erst teilweise umgesetzt. Noch nie seit dessen Inkrafttreten am 1. Januar 2001 wurde die gesetzlich vorgeschriebene detaillierte Statistik über die durchgeführten IVF-Behandlungen veröffentlicht. Dadurch fehlen wichtige Daten, die zusammen mit den ethischen Argumenten in der Diskussion im Vorfeld der Abstimmung über das Stammzellenforschungsgesetz (StFG) vom 28. November 2004 unerlässlich sind. Welche Auswirkungen nämlich die Annahme dieses Gesetzes auf die Praxis der Fortpflanzungsmedizin in der Schweiz hat, ist mit dem spärlichen Zahlenmaterial des Bundes kaum abzuschätzen. Mit Hilfe der FIVNATStatistiken der Schweizerischen Gesellschaft für Reproduktionsmedizin und dem IVF-Register der Bundesrepublik Deutschland lässt sich etwas Licht in die Angelegenheit bringen. Dabei stellen die Autoren fest, dass mit dem Stammzellenforschungsgesetz eine gefährliche Grauzone entsteht, die sich einer Kontrolle von aussen weitgehend entzieht. Nach den vorliegenden Regelungsentwürfen bliebe die Zahl der für die Gewinnung von embryonalen Stammzellen geopferten Embryos nur einem eingeweihten Personenkreis (IVF-Kliniken, Forschung, BAG) vorbehalten.

\section{Zur Ausgangslage}

Die Bundesbehörden haben das Fortpflanzungsmedizingesetz (FMedG) bisher erst teilweise umgesetzt. Nach Art. 11 des FMedG müssen die Kliniken und Praxen den kantonalen Kontrollbehörden jährlich ausführlich über die bei ihnen durchgeführten IVF-Zyklen Bericht erstatten. Insbesondere ist die Zahl und die Art der Behandlungen (Abs. 2, Bst. a), die Zahl der Schwangerschaften und deren Ausgang (Bst. d), die Konservierung und Verwendung von Keimzellen und imprägnierten Eizellen (Bst. e) und die Anzahl der sogenannten überzähligen Embryos (Bst. f) zu melden. Die Bewilligungsbehörden leiten dann die Daten an das Bundesamt für Statistik zur «Auswertung und Veröffentlichung» (Abs. 4) weiter. Nach der Fortpflanzungsmedizinverordnung (FMedV), die ebenfalls am 1. Januar 2001 in Kraft getreten ist, müssen die Kliniken ihren Tätigkeitsbericht bis zum 1. Mai des folgenden Jahres zustellen (Art. 14, Abs. 1). Die kantonalen Aufsichtsbehörden übermitteln dann die anonymisierten Daten dem Bundesamt für Statistik bis zum 1. Juli des betreffenden Jahres zur Auswertung und Veröffentlichung (Art. 14, Abs. 2).

Das Bundesamt für Statistik hat diesen Auftrag bisher nicht erfüllt. Statt dessen versuchte das Bundesamt für Gesundheit in den letzten beiden Jahren, durch Umfragen Zahlenmaterial zusammenzutragen. Die Ergebnisse schlugen sich lediglich in Medienmitteilungen im April 2003 und im Juni 2004 nieder. Demnach sind in der Schweiz in den Jahren 2001 bis 2003 je «rund 200 Embryos» überzählig geworden. Bei der letzten Datenerhebung wurden zwei neue Kategorien erfasst. Zusätzlich zu den «rund 200» überzähligen Embryos im Jahr 2003 kommen weitere «rund 300» Embryos, die lediglich vaginal, d.h. faktisch zum Absterben, transferiert wurden. Erfasst wurden ausserdem diejenigen «rund 200» Embryos, die bereits in der Petrischale aufhörten sich zu entwickeln und abstarben. Als Gründe für die Überzähligkeit werden das schlechte Entwicklungspotential, Willensänderung, Krankheit, Unfall oder Tod der Frau angeführt.

\section{Details zur Praxis der Embryotransfers und der Kryokonservierung}

Die Schweizerische Gesellschaft für Reproduktionsmedizin (SGRM) hat die Statistiken der Jahre 2000-2002 online zugänglich gemacht [1]. Darin werden praktisch alle in der Schweiz durchgeführten IVF-Behandlungen erfasst. Dabei fällt auf, dass sich seit 2001 die Tiefgefrierpraxis in den Kliniken enorm verändert hat. Wurden vor Inkrafttreten des FMedG noch im Jahr 2000 nicht weniger als 816 Embryos tiefgefroren, 
waren es im Jahr 2001 «nur» noch 97. Nach dem FMedG Art. 17, Abs. 3, ist zwar das Tiefgefrieren der Embryos verboten, doch wurde schon in der Botschaft zu diesem Gesetz darauf hingewiesen, dass es Notsituationen geben könne (Überstimulation, Krankheit, Unfall), bei denen zur Rettung des Embryos und zur späteren Herbeiführung einer Schwangerschaft eine Tiefgefrierung möglich sei [2]. Ein Vergleich mit der BRD zeigt aber, dass in der Schweiz im Jahr 2001 in Relation zu den durchgeführten IVF-Zyklen rund zehnmal mehr Embryos kryokonserviert wurden. Somit wurde in den Schweizer Kliniken und Praxen der Begriff der Notsituation wohl stark überstrapaziert (FIVNAT 2001: 97/3147 Zyklen; IVF-Register BRD 1998-2001: 328/184436 Zyklen). Dabei ist zu bedenken, dass jeder tiefgefrorene Embryo früher oder später zu einem überzähligen Embryo werden kann.

Für das Jahr 2003 liegt derzeit noch keine FIVNAT-Statistik vor. Daher muss das Jahr 2002 für einen Vergleich der FIVNAT-Statistik mit den Angaben des BAG herangezogen werden. Das BAG gibt an, rund 200 Embryos seien überzählig geworden. Der FIVNAT-Statistik entnehmen wir, dass im selben Jahr 64 Embryos tiefgefroren wurden, 111 aufgetaut und nicht weniger als 665 Embryos im Rahmen von IVF-Zyklen gar nicht transferiert wurden. Hinzu kommen 2101 Zygoten und Embryos, die nach dem Auftauen ebenfalls nicht transferiert wurden. Aufgrund der dürftigen Angaben des BAG lässt sich nicht mit Sicherheit sagen, ob die Zahl von 200 wirklich plausibel ist. Sicher ist nur, dass sich darunter Embryos befinden müssen, die im Rahmen von IVF-Zyklen und Kryo-Zyklen nicht den gängigen Selektionskriterien entsprachen und daher nicht transferiert wurden.

\section{Anmerkungen zur ethischen Problematik}

Mit Blick auf die eben angeführten Fakten muss beachtet werden, worum es hier eigentlich geht. Mit den Statistiken werden nicht irgendwelche Gegenstände erfasst, sondern es geht um Menschen, deren Existenz in hohem Mass von den Bedingungen im Labor und den Entscheidungen der Reproduktionsmedizin sowie der Eltern abhängt. Die Verschmelzung von Samen- und Eizelle ist die entscheidende Voraussetzung, damit ein neuer Mensch entsteht. Die Existenz jedes geborenen Kindes geht darauf zurück. Die Reproduktionsmedizin macht sich dieses Faktum bei der IVF zunutze. Die befruchtete Eizelle in ihrem frühesten Stadium hat eine ernorme
Entwicklungspotenz hin zu einem erwachsenen Menschen mit einer Lebenserwartung von durchschnittlich etwa 75 Jahren. Ei- und Samenzelle je für sich betrachtet haben lediglich eine Überlebenspotenz von Stunden bzw. Tagen. Wenn wir die menschliche Vernunft und den freien Willen als Auszeichnung des Menschen betrachten, müssen wir den Menschen vom ersten Augenblick der Empfängnis achten, weil diese die notwendige Voraussetzung für deren künftige Entwicklung und Ausübung bildet. Aus der Tatsache, dass der Mensch in der ersten Phase seiner Existenz nur aus einer Zelle besteht, folgert nicht, dass man ihn nicht respektieren müsste.

Indem in der Bundesverfassung der Schutz der Menschenwürde in Art. 7 allgemein und in Art. 119 im Zusammenhang mit seiner Fortpflanzung verankert ist, ist der Staat verpflichtet, jene Massnahmen zu ergreifen, die ausreichenden Schutz gewährleisten. Wie nachfolgend aufgezeigt wird, schützt das StFG die Menschenwürde keineswegs, wie in Art. 1, Abs. 2 behauptet.

\section{Absehbare Entwicklung: Fortpflanzungs- medizin und Stammzellenforschung arbeiten Hand in Hand}

Für die Gewinnung einer einzigen Stammzellinie werden je nach Erfahrung der Teams zwischen sieben und über 30 Embryos benötigt $[3,4]$. Wenn Embryos mit vermindertem Entwicklungspotential verwendet werden, dürfte sich diese Zahl wesentlich erhöhen. Der Bedarf an Embryos allein schon für die Verbesserung des Gewinnungsverfahrens dürfte enorm hoch sein. Das StFG und die Verordnung dazu regeln, wie die benötigten Embryos der Forschung zugeführt werden sollen.

Mit der Auflage, wonach zuerst die Überzähligkeit der Embryos festgestellt werden muss und erst danach das Paar angefragt wird, will der Verordnungsentwurf zum StFG eine Trennung zwischen Fortpflanzungsverfahren und Forschung gewährleisten. (VStFG Art. 2, Abs. 1). Obwohl Art. 6 des StFG ausdrücklich eine Unabhängigkeit zwischen Fortpflanzungsmedizin und Forschungsprojekt vorschreibt, wird diese mit Blick auf die Botschaft zum früheren Art. 11 des Embryonenforschungsgesetzes nicht strikte vollzogen. Fortpflanzungsmediziner können durchaus Stammzellen aus überzähligen Embryos gewinnen, sofern diese nicht aus Behandlungen stammen, die sie selber durchgeführt haben. 
Gemäss FIVNAT-Statistik der SGRM wurden im Jahr 2000, als das FMedG noch nicht in Kraft war, etwa $10 \%$ der Transfers am 4 . Tag und später durchgeführt. Während es im Jahr 2001 keine derartigen Transfers mehr gab, wurde diese Praxis im Jahr 2002 offenbar wieder aufgenommen. Etwa 5\% der Transfers wurden am 4. Tag und später durchgeführt. Durchschnittlich wurden dabei je Zyklus 4,6 Embryos entwickelt. Dabei dürfen doch gemäss Bundesverfassung Art. 119 und FMedG Art. 17, Abs. 1, «nur so viele menschliche Eizellen ausserhalb des Körpers der Frau zu Embryos entwickelt werden, als ihr sofort eingepflanzt werden können» [5]. Transferiert wurden hingegen lediglich durchschnittlich 2,5. Nahezu die Hälfte aller Embryos erlitt folglich den Reagenzglastod bzw. wurde als nicht transferierbar beurteilt. Den Zahlen ist zu entnehmen, dass Embryos auf Vorrat erzeugt werden, damit letztlich eine ausreichende Anzahl übertragen werden kann. Diese Praxis widerspricht mit Sicherheit der ursprünglichen Intention der Bundesverfassung und dem FMedG. Mit Respekt auf das in-vitro-gezeugte Leben dürften nicht mehr Embryos aus imprägnierten Eizellen entwickelt werden, als der Frau sofort verpflanzt werden können.

Der Blastozystentransfer (5. Tag) ist mit einer höheren Implantationsrate verbunden, hat aber auch den Nachteil, dass sich das Geschlechtsverhältnis der geborenen Kinder signifikant auf etwa 1,3 (männlich/weiblich) ändert [6, 7]. Wenn nun Reproduktionsmedizin und Forschung Hand in Hand arbeiten, liegt es nahe, sofern das kantonale Kontrollinstanzen wie im Jahr 2002 durchgehen lassen, dass vermehrt Blastozystentransfers angestrebt werden. Bereits bei zwei morphologisch einwandfreien Blastozysten und erst recht bei drei ist das Mehrlingsrisiko viel höher als bei Transfers, die im achtzelligen Stadium durchgeführt werden. Sofern das Paar zuvor zu verstehen gab, dass es nicht mehr als zwei oder nur einen Embryo transferieren lassen möchte, werden die anderen automatisch überzählig [8]. Sie könnten ohne Tiefgefrierung direkt der Stammzellengewinnung zugeführt werden, sofern die Eltern einverstanden sind. Da die Wahrscheinlichkeit für die Erzeugung einer Stammzellinie mit der Entwicklungspotenz des Embryos gekoppelt ist, wären solche Embryos für die Forschung weit «interessanter» als tiefgefrorene oder aufgetaute mehrzellige Embryos mit häufig verminderter Entwicklungspotenz.

\section{Die Feststellung der Überzähligkeit des Embryos ist gleichbedeutend mit seinem Todesurteil}

Das Stammzellenforschungsgesetz verändert nun die Situation in der Reproduktionsmedizin ganz entscheidend. Die Entwicklung des Embryos in der Petrischale ist nicht mehr für einen, sondern für zwei Zwecke offen: Für den Transfer in die Gebärmutter und die Verwendung zur Stammzellengewinnung, falls er überzählig werden sollte. Auch wenn die Embryos ursprünglich zum Zweck der Fortpflanzung gezeugt wurden, lässt sich der zweite nun hinzukommende allfällige Verwendungszweck nicht mehr aus dem Arbeitsfeld des in der assistierten Reproduktionsmedizin tätigen Personals ausklammern. Die Embryos entwickeln sich in vitro auf einen Zeitpunkt von grösster Tragweite hin: der Feststellung seiner Transferierbarkeit bzw. seiner Überzähligkeit.

Indem das StFG die Feststellung der Überzähligkeit dem medizinischen Personal überträgt, welches erst danach das betroffene Paar über den Verwendungszweck der überzähligen Embryos anfragt, wird eine gefährliche Grauzone geschaffen. Wie wird denn genau definiert, was ein Embryo mit schlechtem Entwicklungspotential ist?

Die Meinungen gehen bei den Fachleuten auseinander. Sicherheitshalber werden die morphologisch intakten Embryos transferiert, sofern aber nur ein solcher vorhanden ist, pflegen etliche Kliniken durchaus einen Embryo mitzutransferieren, der ein vermeintlich schlechtes Entwicklungspotential hat. Damit werden Schwangerschaften und auch Geburten von gesunden Babys erzielt [9].

\section{Nur scheinbare Transparenz der Bundesbehörden}

Die VStFG enthält einige Bestimmungen, wonach bestimmte Daten veröffentlicht werden müssen. Diese Transparenz erweist sich bei genauerem Hinsehen nur als schwacher Lichtstrahl in die Dunkelkammer der Forschung mit embryonalen Stammzellen. Das Bundesamt für Gesundheit muss Art. 28, Abs. 3, gemäss lediglich die Anzahl der embryonalen Stammzellinien, die im Rahmen eines Forschungsprojektes erzeugt wurden, veröffentlichen. Die Anzahl der 
dafür verwendeten überzähligen Embryos wird zwar gemäss Art. 23, Bst. a, erfasst, muss aber nicht bekanntgegeben werden! Sofern sich am Entwurf der VStFG nichts ändert, würde der Schweizer Stimmbürger nach Annahme des StFG niemals erfahren, wie viele Embryos zur Stammzellengewinnung in der Schweiz geopfert werden.

\section{Schlussfolgerungen}

Mit der Annahme des StFG würde in den IVF-Kliniken und Praxen der Schweiz eine Grauzone geschaffen, die sich der Kontrolle durch Bund und Kantone entzieht. Der Forschung würde Zutritt in diese Grauzone verschafft, so dass Forschung und Reproduktionsmedizin ausser Kontrolle geraten. Zudem bestünde die Gefahr, dass sich diese Grauzone mehr und mehr vergrössert. Die Forschung könnte sich mit Embryos, die nicht als transferierbar beurteilt werden, bedienen. Die Feststellung der Überzähligkeit wäre allein dem medizinischen Personal übertragen. Die Paare hätten lediglich zu entscheiden, ob ihre Embryos dem sofortigen Reagensglastod oder dem Tod durch Stammzellengewinnung überlassen werden sollen. Den Beweis, dass die Fortpflanzungsmedizin sich wirklich im Rahmen der Bundesverfassung und des FMedG bewegt, sind die Kontrollorgane von Bund und Kantonen bisher schuldig geblieben. Das StFG und dessen Verordnungsentwurf mit insgesamt 63 Artikeln können nicht darüber hinwegtäuschen, dass dieses Werk nicht dem Schutz der Menschenwürde in einem äusserst sensiblen Bereich dient, sondern einem überstrapazierten Freiheitsverständnis der Forschung. Diese sollte sich vielmehr den adulten Stammzellen zuwenden, mit denen jetzt schon Therapieerfolge erzielt werden und die nach gegenwärtigem Wissensstand für die Zukunft sehr vielversprechend sind [10-14]. Zur erwähnten ethischen Problematik bei den embryonalen Stammzellen kommt hinzu, dass deren charakteristische Neigung zur Tumorbildung sowie die $\mathrm{zu}$ erwartende Immunabwehr im Körper des Empfängers von Transplantaten derzeit und auch in absehbarer Zukunft keinen Therapieeinsatz erlauben $[15,16]$. Mit Blick auf die Anwendungsmöglichkeiten der adulten Stammzellen, die u.U. dem Patienten sogar selber entnommen, modifiziert und zurücktransplantiert werden können, drängt sich die Frage auf, ob dieses verbissene Festhalten der Forschung und vieler Politiker an den embryonalen Stammzellen nicht geradezu Ressourcen verschwendet, die besser in die Forschung mit adulten Stammzellen mit weit schnelleren und reellen Therapieaussichten investiert werden sollten. Dies betonten Wissenschaftler an einer Sozialethikertagung in Mönchengladbach (Antworten auf Fragen von morgen: Welche Stellung hat der Mensch in der modernen Biomedizin?) vom Mai 2004. Sie gingen auf die gravierenden Nachteile der embryonalen Stammzellen ein, die dazu führen werden, dass die Pharmaindustrie künftig in die adulte statt in die embryonale Stammzellenforschung investieren werde. Prof. Hans Edgar Reis: «Die Industrie wird mit Sicherheit die nebenwirkungsärmste Form wählen». Dies nicht aus ethischen Gründen, sondern um «Regresse zu vermeiden» [17].

\section{Literatur}

1 Siehe Webseite der Schweizerischen Gesellschaft für Reproduktionsmedizin: www.sgrm.org. Dort sind die Statistiken für die Jahre 2000-2002 online abrufbar.

2 Botschaft über die Volksinitiative «zum Schutz des Menschen vor Manipulationen in der Fortpflanzungstechnologie» und zu einem Bundesgesetz über die medizinisch unterstützte Fortpflanzung (FMedG) S. 62.

3 Thomson JA, Itskovitz J, Shapira SS, Waknitz MA, Swiergiel JJ, Marshall VS, et al. Embryonic stemcell lines derived from human blastocysts. Science 1998;282:1145-7.

4 Lanzendorf SE, Boyd CA, Wright DL, Muasher SJ, Oehninger SC, Hodgen GD. The use of gametes obtained from anonymous donors for the production of human embryonic stem cell (ESC) lines. Fertil Steril 2000;74(Suppl 1):S16-S17.

5 Botschaft über die FMF-Initiative und zum FMedG S. 62. Wörtlich wird in der Botschaft Art. 17 des FMedG wie folgt interpretiert: «Im Lichte von Artikel 24novies Abs. 2 Bst. c BV verbietet Artikel 17 Abs. 1, mehr imprägnierte Eizellen zu Embryos zu entwickeln, als innerhalb eines Monatszyklus der Frau für die Herbeiführung einer Schwangerschaft erforderlich ist. Aufgrund von Anregungen im Vernehmlassungsverfahren wird die Maximalzahl auf drei festgelegt.»

6 Kausche A, Jones GM, Trounson AO, Figueiredo F, MacLachlan V, Lolatgis N. Sex ratio and birth weights of infants born as a result of blastocyst transfers compared with early cleavage stage embryo transfers. Fertil Steril 2001;76:688-93.

7 Jun SH, Milki M. A higher male to female sex ratio is seen with blastocyst transfer compared to cleavage stage transfer. Fertil Steril 2004; 81(Suppl 3):29.

8 Nach Art 2 Bst. b des StFG wird ein überzähliger Embryo wie folgt definiert: «Im Rahmen der In-vitro-Fertilisation erzeugter Embryo, der nicht zur Herbeiführung einer Schwangerschaft verwendet werden kann und deshalb keine Überlebenschance hat.» 
9 Glasen K, Delbeke L, De Loecker P, Peeters K, Punjabi U, Van Mulders $H$. The total embryo score is a better predictor of IVF outcome than is the number of transferred embryos. 11. World Congress on Human Repraduction 1.-4. Juni 2002, Abstract O-073. www.rbmonline.com/ 4DCGI/Article/Article?38\%091\%09=\%20666\%09.

10 Wellert KC, Meyer GP, Lotz J, Ringes-Lichtenberg S, Lippolt P, Breidenbach C, et al. Intracoronary autologous bone-marrow cell transfer after myocardial infarction: The BOOST Randomised Contralled Clinical Trial. Lancet 2004;364:141-8.

11 Jang Y-Y, Collector MI, Baylin SB, Diehl AM, Sharkis SJ. Hematopoietic stem cells convert into liver cells within days without fusion: Nat Cell Biol 2004;6:532-9.

12 Joannides A, Gaughwin P, Schwiening C, Majed $\mathrm{H}$, Sterling J, Compston A, et al. Efficient generation of neural precursors from adult human skin: astrocytes promote neuragenesis from skinderived stem cells. Lancet 2004;362:172-8.
13 Dyer O. «Bubble Baby» lives normal life after gene therapy. Br Med J 2002;324:872.

14 Erickson GR, Gimble JM, Franklin DM, Rice HE, Awad H, Guilak F. Chondrogenic potential of adipose tissue-derived stramal cells in vitra and in vive. BBRC 2002;290:763-9.

15 Erdö F, Bührle C, Blunk J, Hoehn M, Xia Y, Fleischmann B, et al. Host-dependent tumorigenesis of embryonic stem cell transplantation in experimental stroke. J Cereb Blood Flow Metab 2003;23:780-5.

16 Freed CR, Leehey MA, Zawada M, Bjugstad K, Thompson L, Breeze RE. Do patients with Parkinson's disease benefit from embryonic dopamine cell transplantation? J Neurol 2003; 250(Suppl 3):44-6.

17 Rehder S. Antworten auf Fragen von morgen: Die Tagespost 6. Mai 2004;57(54):3. www.dietagespost.com/Archiv/titel_anzeige.asp?ID=8748. 